\title{
Extraction of step-repulsion strengths from terrace width distributions: statistical and analytical considerations
}

\author{
H.L. Richards ${ }^{\text {a }}$, S.D. Cohen ${ }^{\text {a }}$, T.L. Einstein ${ }^{\mathrm{a}, *}$, M. Giesen ${ }^{\mathrm{b}}$ \\ a Department of Physics, University of Maryland, College Park, MD 20742-4111, USA \\ ${ }^{\mathrm{b}}$ Institut für Grenzflächenforschung und Vakuumphysik, Forschungszentrum Jülich, 52425 Jülich, Germany
}

Received 1 November 1999; accepted for publication 17 January 2000

\begin{abstract}
Recently it has been recognized that the so-called generalized Wigner distribution may provide at least as good a description of terrace width distributions (TWDs) on vicinal surfaces as the standard Gaussian fit and is particularly applicable for weak repulsions between steps, where the latter fails. Subsequent applications to vicinal copper surfaces at various temperatures confirmed the serviceability of the new analysis procedure but raised some theoretical questions. Here we address these issues using analytical, numerical, and statistical methods. We propose an extension of the generalized Wigner distribution to a two-parameter fit that allows the terrace widths to be scaled by an optimum effective mean width. We discuss quantitatively the approach of a Wigner distribution to a Gaussian form for strong repulsions, how errors in normalization or mean affect the deduced interaction, and how optimally to extract the interaction from the variance and mean of the TWD. We show that correlations reduce by two orders of magnitude the number of independent measurements in a typical scanning tunneling microscopy image. We also discuss the effect of the discreteness ('quantization') of terrace widths, finding that for high misorientation (small mean width) the standard continuum analysis gives faulty estimates of step interactions. (C) 2000 Elsevier Science B.V. All rights reserved.
\end{abstract}

Keywords: Copper; Equilibrium thermodynamics and statistical mechanics; Stepped single crystal surfaces; Surface structure, morphology, roughness, and topography; Vicinal single crystal surfaces

\section{Introduction}

During the last decade a number of researchers have used atomic-scale microscopy to make quantitative experimental measurements of the terrace width distribution (TWD) of vicinal surfaces. To understand the data - and, especially, to extract the strength of the interaction between the steps they have fit the TWDs with Gaussians (or in cases of no apparent energetic repulsion, with free-

* Corresponding author. Fax: + 1-301-314-9465.

E-mail address: einstein@physics.umd.edu (T.L. Einstein) fermion distributions). Recently there has been a significant improvement in the theoretical understanding of interacting steps on vicinal surfaces: as an example of a fluctuation phenomenon, they should be described by certain universal features related to random-matrix theory. In particular, the TWD should be well describable in terms of a generalized form of the distribution surmised by Wigner to describe some special cases of interactions [1].

In a recent paper [2], hereafter GE, TWDs of various vicinal copper surfaces were analyzed using both the traditional Gaussian approach and the 
generalized Wigner surmise. Many conclusions were noted in passing about the relative merits and sensitivities of these two approaches. The goal of this paper is to provide supporting details together with new results and approaches that should aid in the interpretation and analysis of experimental TWDs. We explore the relationship between the Wigner form of TWDs and the Gaussian. We discuss several statistical considerations that should be taken into account. The many issues treated by this paper arose during the course of analyzing experimental data in GE.

This paper is organized as follows. Section 2 reviews the TWD derived from the generalized Wigner surmise and presents some practical new approximations derived from series expansions. In particular, we provide what we believe is the best simple expression [Eq. (7)] to deduce the step-step repulsion strength from the variance of the TWD. Section 3 deals with the approach of the generalized Wigner distribution to the form of the Gaussian for strong step-step repulsions. While this behavior had been recognized earlier, we now characterize it quantitatively. In Section 4, we contend with a recurring theme in GE: the error generated by uncertainty in the mean of the distribution. Experimentalists had the belief that Gaussian fits of the data are more forgiving of such errors than are Wigner fits. We study this notion quantitatively by checking, for both distributions, the effect of perturbations in normalization and in mean by fitting deliberately misnormed or displaced data. The results of arguably greatest interest to experimentalists are in Section 5. We describe an extension of our proposed analysis scheme for TWDs for which the first moment of the data does not conform well to the apparent mean. We propose treating the generalized Wigner distribution as a two-parameter function: in addition to the exponent $\varrho$, the value of the effective mean (which scales the terrace widths; cf. Section 2) is adjusted simultaneously in the nonlinear least-squares fit. This procedure makes little difference for the 'good' data reported in GE, but can have significant effect on 'poorer' data glossed over in that paper. We present both graphical illustrations and thorough tabulations for the extensive data for vicinal copper discussed in GE.
We also apply the Wigner distribution to recently published data for vicinal $\operatorname{Pt}(110)$. Sections 6 and 7 offer a pair of warnings regarding how the discreteness of the terrace widths and the limited size of the sample, respectively, can confound the analysis. In the former case, for the range of interaction strengths found in physical systems, discreteness becomes problematic for high misorientations, when the mean terrace width drops to just a few lattice spacings. In the latter case, we observe that statistical fluctuations due to the typical size might well account for some of the data sets labeled as 'poor', rather than some system contaminant or measurement flaw. The Conclusion summarizes the current state of our understanding.

\section{Generalized Wigner surmise: recap of key formulas and new results from series expansion}

As has been discussed extensively before [1,2], a new idea from random-matrix theory $[3,4]$ is that fluctuations should exhibit certain universal behavior. According to the so-called Wigner surmise, the distribution of fluctuations can be approximated by [1]

$P_{Q^{(s)}=a_{Q}} s^{\varrho} \exp \left(-b_{\varrho} s^{2}\right)$

where $s=l /\langle l\rangle, l$ being the terrace width, and the constants $b_{\varrho}$ and $a_{\varrho}$ are given by:

$$
\begin{aligned}
b_{\varrho} & =\left[\frac{\Gamma((\varrho+2) / 2)}{\Gamma((\varrho+1) / 2)}\right]^{2} \\
& \approx\left(\frac{\varrho+1}{2}\right)\left[1-\frac{1}{2}(\varrho+1)^{-1}+\frac{1}{8}(\varrho+1)^{-2}\right] \\
& =\frac{\varrho}{2}+\frac{1}{4}+\frac{1}{16(\varrho+1)}
\end{aligned}
$$

and

$a_{\varrho}=\frac{2 b_{\varrho}^{(\varrho+1) / 2}}{\Gamma((\varrho+1) / 2)}=\frac{2[\Gamma((\varrho+2) / 2)]^{\varrho+1}}{[\Gamma((\varrho+1) / 2)]^{\varrho+2}}$,

respectively. For brevity, we refer hereafter to this set of formulas as the CGWD (continuum generalized Wigner distribution). The CGWD can be 
derived in a more transparent fashion from a mean-field approximation [5].

The approximate result in Eq. (2), derived in Appendix A by asymptotic expansion, is new. It is consistent with Eq. (9) of GE in the neighborhood of $\varrho=4$; it is within $0.2 \%$ of the exact $b \varrho$ as calculated using gamma functions at $\varrho=2$ and is within $0.05 \%$ of $b_{\varrho}$ by $\varrho=4$.

Experimentally, a TWD is typically characterized by its variance $\sigma^{2}$. In principle $\sigma^{2}$ might be determined directly from the second moment of the TWD, but there is concern that this approach does not adequately minimize noise in the data, an issue we shall revisit in Section 7. Thus, in practice, TWDs are fit to smooth functions; Gaussians are typically chosen, not just for their simplicity but because their use can be justified readily in the limit of strong elastic repulsion between steps. The variance of the TWD is then approximated by the variance $\sigma_{\mathrm{G}}^{2}$ of the fitted Gaussian. We argue here and in GE that the CGWD given in Eq. (1) is scarcely more complicated than a Gaussian but provides a better accounting of the variance. For strong step repulsions, the variance of the fitted Gaussian is usually not very different from the variance $\sigma_{\mathrm{w}}^{2}$ of a CGWD, as is discussed more quantitatively in Section 3. For weak repulsions, however, it is well known that the TWD becomes too skewed to allow a satisfactory fit to a Gaussian. Experimentalists finding themselves in this predicament have been stymied on how to proceed quantitatively [6,7]. Significantly, a Gaussian fit to a TWD with non-negligible skewness cannot even be expected to have the correct mean; the consequences of this fact are dealt with in much of the reminder of this paper.

For the CGWD, the variance can be expressed simply in terms of $b_{\varrho}$. We can use Eq. (2) to obtain

$$
\begin{aligned}
\sigma_{\mathrm{w}}^{2} & =\frac{\varrho+1}{2 b_{\varrho}}-1 \\
& \approx \frac{1}{2}(\varrho+1)^{-1}+\frac{1}{8}(\varrho+1)^{-2}
\end{aligned}
$$

for large values of $\varrho$ (e.g. $\sigma^{2}$ is overestimated by ca. $0.5 \%$ at $\varrho=4$ but just $0.1 \%$ at $\varrho=10$ ).

The usual goal in an experiment is to extract the magnitude $A$ of the elastic repulsion between steps, perpendicular to the step direction, given by $A / l^{2}$. All standard analysis procedures make a continuum approximation in the direction along the steps (perpendicular to the 'upstairs' direction); thereafter, $A$ appears only in the form of a dimensionless interaction strength $\tilde{A} \equiv A \tilde{\beta}\left(k_{\mathrm{B}} T\right)^{-2}$, where $\tilde{\beta}$ is the step stiffness. In this conceptualization $\varrho$ is related to $\tilde{A}$ by the equation:

$\tilde{A}_{\mathrm{W}}=\frac{\varrho(\varrho-2)}{4}$

which follows from mapping this problem onto the Sutherland Hamiltonian [8]. The subscript W provides a reminder that this estimate of $\tilde{A}$ is based on the CGWD. Eq. (4b) can be solved for $\varrho$, which in turn can be inserted into Eq. (5) to provide a good estimate for $\tilde{A}_{\mathrm{w}}$. However, a much better estimate of $\tilde{A}_{\mathrm{w}}$ - visually indistinguishable from the exact value on a standard-resolution graph - comes from performing a reversion of series of a higher-order version of Eq. (4b) to yield $\varrho$ as a function of $\sigma^{2}$,

$\varrho \approx \frac{1}{2}\left(\sigma^{2}\right)^{-1}\left\{1-\frac{3}{2}(\sigma)^{2}-\frac{3}{4}\left(\sigma^{2}\right)^{2}+\frac{7}{24}\left(\sigma^{2}\right)^{3}\right\}$,

and then inserting this result into Eq. (5):

$\tilde{A}_{\mathrm{W}} \approx \frac{1}{16}\left[\left(\sigma^{2}\right)^{-2}-7\left(\sigma^{2}\right)^{-1}+\frac{27}{4}+\frac{35}{6} \sigma^{2}\right]$.

Eq. (7) should prove quite useful in analyzing data, since it provides an excellent value for $\tilde{A}$ as a function of the variance of the TWD, assuming the validity of the CGWD description. We caution that all four terms must be kept in order to obtain a good estimate of $\tilde{A}$ from Eq. (7). We also warn that, as discussed in Section 6, the effects of discreteness may lead to inconsistencies with this estimate for highly misoriented vicinal surfaces. 
Table 1

Tabulation of the results of fitting data from various vicinal surfaces of copper to a Gaussian with three parameters (labeled by subscript $G$ ) and to Wigner distributions with one or with two adjustable parameters (labeled by subscripts 1 and 2 , respectively) ${ }^{\mathrm{a}}$

\begin{tabular}{|c|c|c|c|c|c|c|c|c|c|c|c|}
\hline$T$ & Qual & $\sigma_{\mathrm{G}}$ & $100 \chi_{\mathrm{G}}^{2}$ & $\varrho_{1}$ & $\sigma_{1}$ & $100 \chi_{1}^{2}$ & $\varrho_{2}$ & $\sigma_{2}$ & $100 \chi_{2}^{2}$ & $\Delta \mu$ & $\sigma_{\mathrm{dir}}$ \\
\hline \multicolumn{12}{|c|}{$(1,1,7)$} \\
\hline $\begin{array}{l}298 \\
(1,1,\end{array}$ & \multicolumn{3}{|c|}{$(1,1,13)$} & $11.0(6)$ & $0.21(1)$ & 0.32 & $11.0(6)$ & $0.21(1)$ & 0.35 & -0.00 & 0.23 \\
\hline 285 & + & 0.19 (2) & 1.31 & $9.7(12)$ & $0.22(1)$ & 2.21 & $10.0(8)$ & 0.21 & 1.16 & 0.04 (1) & 0.25 \\
\hline 300 & + & $0.25(1)$ & 0.73 & $6.4(5)$ & $0.26(1)$ & 0.78 & $6.5(3)$ & $0.26(1)$ & 0.36 & 0.04 (1) & 0.28 \\
\hline 303 & 0 & $0.26(1)$ & 0.58 & $5.7(6)$ & $0.28(1)$ & 1.00 & $5.9(3)$ & 0.27 & 0.31 & 0.05 (1) & 0.36 \\
\hline 320 & + & $0.27(2)$ & 0.61 & $5.2(4)$ & $0.29(1)$ & 0.79 & $5.3(4)$ & 0.29 (1) & 0.39 & 0.04 (1) & 0.33 \\
\hline 326 & - & $0.27(2)$ & 1.52 & $2.8(6)$ & $0.38(3)$ & 3.34 & $3.5(4)$ & 0.34 & 1.44 & $0.13(3)$ & 0.45 \\
\hline 330 & $-/ 0$ & $0.28(2)$ & 1.86 & $3.9(6)$ & $0.33(1)$ & 1.80 & $4.2(4)$ & $0.32(2)$ & 1.16 & $0.06(2)$ & 0.35 \\
\hline 338 & + & $0.25(1)$ & 0.52 & $5.3(6)$ & $0.29(1)$ & 1.13 & $5.6(3)$ & 0.28 (1) & 0.31 & 0.06 (1) & 0.34 \\
\hline 348 & + & 0.27 (1) & 0.88 & $4.4(5)$ & $0.31(1)$ & 1.59 & $4.8(4)$ & $0.30(1)$ & 0.57 & 0.07 (1) & 0.36 \\
\hline 350 & $0 /+$ & $0.27(1)$ & 0.16 & $5.0(4)$ & $0.29(2)$ & 0.89 & $5.1(3)$ & 0.29 & 0.23 & 0.06 (1) & 0.40 \\
\hline 358 & 0 & $0.21(2)$ & 0.79 & $5.6(10)$ & 0.28 & 2.71 & $6.8(6)$ & $0.26(1)$ & 0.87 & 0.09 (1) & 0.38 \\
\hline 368 & - & $0.25(2)$ & 1.77 & $3.1(5)$ & $0.36(3)$ & 2.89 & $3.9(4)$ & 0.33 (1) & 1.57 & $0.12(2)$ & 0.45 \\
\hline 378 & - & $0.30(4)$ & 3.52 & $2.5(7)$ & $0.39(3)$ & 4.20 & $3.0(5)$ & $0.36(3)$ & 2.48 & $0.14(3)$ & 0.46 \\
\hline \multicolumn{12}{|c|}{$(1,1,19)$} \\
\hline 290 & - & $0.40(4)$ & 3.74 & $2.7(4)$ & $0.38(2)$ & 2.33 & $2.7(5)$ & $0.38(2)$ & 2.49 & $-0.01(3)$ & 0.34 \\
\hline 300 & $-/ 0$ & $0.24(2)$ & 2.94 & $3.1(6)$ & $0.36(4)$ & 5.09 & $4.1(6)$ & $0.32(2)$ & 2.69 & $0.12(3)$ & 0.39 \\
\hline 308 & 0 & $0.31(1)$ & 0.71 & $4.4(3)$ & $0.31(1)$ & 0.32 & $4.3(2)$ & 0.31 & 0.30 & 0.01 (1) & 0.30 \\
\hline 320 & + & 0.25 (1) & 0.54 & $6.7(3)$ & $0.26(1)$ & 0.36 & $6.7(2)$ & $0.26(1)$ & 0.23 & 0.02 (1) & 0.27 \\
\hline 360 & + & $0.27(2)$ & 1.74 & $5.7(5)$ & $0.28(1)$ & 0.84 & $5.8(3)$ & 0.28 (1) & 0.64 & 0.03 (1) & 0.29 \\
\hline 370 & - & $0.30(3)$ & 4.20 & $4.0(7)$ & $0.32(3)$ & 3.30 & $4.3(6)$ & $0.31(1)$ & 2.71 & $0.06(2)$ & 0.31 \\
\hline \multicolumn{12}{|c|}{$(11,7,7)$} \\
\hline 296 & + & $0.26(2)$ & 0.55 & $5.7(4)$ & $0.28(1)$ & 0.58 & $5.8(3)$ & 0.28 & 0.27 & 0.04 (1) & 0.30 \\
\hline 301 & + & $0.27(2)$ & 0.80 & $6.0(4)$ & $0.27(1)$ & 0.47 & $6.0(5)$ & 0.27 (1) & 0.46 & $0.01(2)$ & 0.28 \\
\hline 306 & + & $0.28(2)$ & 0.36 & $4.8(3)$ & $0.30(2)$ & 0.48 & $4.9(2)$ & 0.30 (1) & 0.25 & 0.04 (1) & 0.36 \\
\hline 323 & + & 0.29 (1) & 0.20 & $5.0(1)$ & $0.29(1)$ & 0.10 & $5.0(2)$ & 0.29 & 0.10 & 0.00 (1) & 0.31 \\
\hline \multicolumn{12}{|c|}{$(19,17,17)$} \\
\hline 305 & - & $0.23(2)$ & 2.77 & $5.3(7)$ & $0.29(2)$ & 3.62 & $6.3(7)$ & $0.27(2)$ & 2.32 & 0.08 & 0.33 \\
\hline 313 & - & $0.25(2)$ & 2.29 & $4.3(5)$ & $0.32(1)$ & 3.15 & $5.0(4)$ & $0.29(2)$ & 1.90 & 0.09 (1) & 0.38 \\
\hline 333 & $-/ 0$ & $0.34(2)$ & 1.19 & $3.3(3)$ & $0.35(1)$ & 0.90 & $3.3(3)$ & 0.35 (1) & 0.82 & $0.03(2)$ & 0.37 \\
\hline 353 & $-/ 0$ & $0.31(2)$ & 1.11 & $4.0(3)$ & $0.32(1)$ & 0.79 & $4.1(2)$ & $0.32(1)$ & 0.71 & $0.03(2)$ & 0.33 \\
\hline \multicolumn{12}{|c|}{$(23,21,21)$} \\
\hline 318 & $-/ 0$ & $0.24(1)$ & 0.92 & $7.1(4)$ & $0.25(2)$ & 1.10 & $7.2(4)$ & $0.25(1)$ & 0.74 & 0.04 (1) & 0.33 \\
\hline 328 & 0 & 0.29 (1) & 1.06 & $5.3(3)$ & 0.29 (1) & 1.05 & $5.4(3)$ & 0.29 (1) & 1.01 & $-0.01(1)$ & 0.31 \\
\hline
\end{tabular}

${ }^{a}$ The temperature in Kelvin is given in the first column and the qualitative characterization ( + for good, 0 for fair, - for poor) in the second. The final column, labeled $\Delta \mu$, indicates how much the mean (or first moment) computed directly from the data exceeds the optimal mean obtained via the second parameter in the two-parameter Wigner fit; using the notation of Eq. (13), we have $\Delta \mu=\alpha^{-1}-1=1-\alpha$. Motivated by the discussion of Section 7, we include in the final column the standard deviation $\sigma_{\text {dir }}$ evaluated directly from the normalized (and adjusted to unit mean) histogram data

\section{Gaussian fits of the generalized Wigner distribution}

A characteristic feature of the CGWD is that as $\varrho$ becomes larger, the curve can be better approximated by a Gaussian. This feature should be expected, since it is accepted that TWDs for strong repulsions are well described by Gaussians. We quantitatively assessed the degree of agreement. One measure is the $\chi^{2}$ of a fit of the 
CGWD to a Gaussian form (with the three parameters - peak position, prefactor, and standard deviation - as adjustable parameters). We find that this measure of the fit improves exponentially with increasing $\varrho$. (Specifically, $0.012144 \exp (-0.5249 \varrho$ ) provides a close upper bound of $\chi^{2}$ for $\varrho>1$.) A second and more useful measure is the relative difference of the standard deviation $\sigma_{\mathrm{G}}$ of the fitted Gaussian from the actual standard deviation $\sigma_{\mathrm{w}}$ of the CGWD, given by the square root of the second moment of the CGWD about its mean of unity. Using Eq. (2) we find that this relative difference is well described by the formula:

$\frac{\sigma_{\mathrm{G}}}{\sigma_{\mathrm{W}}}-1 \equiv \frac{\sigma_{\mathrm{G}}}{\sqrt{\mu_{2}^{\prime}-1}}-1 \approx \frac{0.0568}{\varrho}-\frac{0.0138}{\varrho^{2}}$,

where the expression for the second moment of the TWD with respect to the origin, $\mu_{2}^{\prime}$, is given explicitly as Eq. (11) of GE or Eq. (8) of EP. Thus, at the calibration point for repulsive interactions ( $\varrho=4$, for which an exact solution exists) the agreement is ca. $1 \%$, and improves monotonically with increasing $\varrho$. For this range $(\rho \geqslant 4)$ differences between estimates of $\tilde{A}$ obtained from CGWD and the various Gaussian fit methods are predominantly due to different philosophies of extracting $\tilde{A}$ from $\sigma$ rather than from differences in the fitting methods.

As discussed at length in EP and GE, there are several distinct theories for extracting the dimensionless interaction strength $\tilde{A}$ from $\sigma_{\mathrm{G}}$. Monte Carlo calculations [9] indicate that the CGWD provides an excellent estimate of $\tilde{A}$ over the range of physical values of this repulsion, as well as for stronger values. Thus, as remarked at the end of the previous section, it is the wisest strategy to use Eq. (7) to estimate $\tilde{A}$ from $\sigma$ deduced from the TWD rather than to use the predictions of one of the Gaussian approximations discussed in Table 1 of GE.

\section{Effects of perturbed normalization or mean}

The CGWD is a normalized TWD with unit mean. In GE, the mean was determined straightforwardly from the first moment. The independent variable (the terrace width) was then scaled by this value, and the distribution normalized. In the course of analyzing TWDs, it became obvious that the normalization of the data sets by total area (that is, the zeroth moment) and first moment provides qualitative agreement with the CGWD that is, the 'best fit' CGWD produces a skew distribution that roughly matches the TWD - but it does not match closely enough to reproduce the correct peak position. In order to motivate the more satisfactory treatment of experimental TWDs in Section 4, in this section we discuss the effects of perturbations of the mean step separation and of the normalization on the variables important for extracting interaction strengths ( $\sigma$ for a Gaussian fit and $\varrho$ for a Wigner fit). Such perturbations might arise in experimental data either due to statistical fluctuations or due to physical causes, such as perturbations of the step-step interaction potential $A / l^{2}$ or an incomplete equilibration of the vicinal surface.

To this end, we created an ideal data set by sampling the appropriate distributions at regular intervals. This ideal set was then perturbed by various factors not exceeding $15 \%$, either by shifting the mean or by scaling each point to increase the area under the curve. These perturbed sets were then fit as in GE, by normalized fitting functions with unit mean. Since the true value of $\varrho$ or $\sigma$ for our ideal data set is known, it is easy to determine the error due to the perturbations. In some cases, the errors behave in complicated ways.

In the equations, $\Delta \sigma$ is the fitted value of $\sigma$ minus the known value of $\sigma$ (and similarly for $\Delta \varrho$ ); $\Delta \mu_{0}$ (or $\Delta \mu_{1}$ ) indicates how much the area under (or the first moment of) the constructed curve exceeds the 'proper value'. [Moments about the origin are defined in Eq. (10) of GE. Here for convenience -since we are interested only in differences - we neglect the primes.] The effect of errors in normalization can be described rather simply. The fitted (normalized) curve becomes narrower as the area under the raw curve increases. For a Gaussian, the fractional change in $\sigma$ is approximately linear in the fractional error of the integrated TWD, with a prefactor ca. 2/3:

$\Delta \sigma /\left.\sigma\right|_{\sigma=0.30}=-0.68 \Delta \mu_{0}+0.81\left(\Delta \mu_{0}\right)^{2}$

The coefficients in Eq. (9) are insensitive to the 
value of $\sigma$ : if the standard deviation of the raw curve is reduced from 0.30 to 0.20 , the linear coefficient is unchanged, while the quadratic coefficient is reduced slightly to 0.80 . For the CGWD, the fit is even more nearly linear:

$\Delta \varrho /\left.\varrho\right|_{\varrho}=4.0=1.38 \Delta \mu_{0}$.

Again increased area leads to an effectively sharper distribution. The linear coefficient is nearly double that in Eq. (10), as one might expect from Eq. (13) of GE. This coefficient again is insensitive to the value of $\varrho$ of the raw distribution: for $\varrho=$ 7.0 it dips slightly to 1.37 .

Errors in the mean of the distribution create errors in the fit that are not so easy to describe. The changes in the fitted parameters are quadratic rather than linear in $\Delta \mu_{1}$, and the coefficients depend strongly on the value of $\sigma$ or $\varrho$ of the raw distribution.

For Gaussians, we find that the following expression provides a good approximation for standard deviations between 0.2 and 0.4 (corresponding to $1.5<\varrho<9$ ):

$\frac{\Delta \sigma}{\sigma} \approx \frac{1}{2}\left(\frac{\Delta \mu_{1}}{\sigma}\right)^{2}$.

Appendix B provides an analytic derivation of this approximation as the leading-order term in an expansion of the appropriate Gaussian integral. Eq. (11) can also be generated from straightforward fitting of numerical data. ${ }^{1}$

Thus, as might be expected since the Gaussian is symmetric about its peak, the error is insensitive to the sign of the error in the mean of the raw distribution. The fitted distribution is broader than the raw one, with the fractional error of the fitted $\sigma$ dependent roughly on the 'fractional error' (with respect to $\sigma$ ) of the first moment, that is, increasing as the distribution becomes sharper.

Since the Wigner distribution is not symmetric about its peak, the corresponding error in fitting an off-center CGWD by a properly centered CGWD should not depend purely quadratically on $\Delta \mu_{1}$. Indeed, we find over the range $1<\varrho<8$

\footnotetext{
${ }^{1}$ In this process, one can generate the result $\Delta \sigma / \sigma=$ $0.486 \sigma^{-2.05}\left(\Delta \mu_{1}\right)^{2}$, which is numerically superior to Eq. (11) but does not satisfy proper dimensional behavior.
}

that an excellent approximation is:

$$
\frac{\Delta \varrho}{\varrho}=(0.3 \varrho-3.0) \Delta \mu_{1}+(-2.0 \varrho+0.4)\left(\Delta \mu_{1}\right)^{2} \text {. }
$$

Analogous to the previous result for $\sigma$, the fractional error of $\varrho$ has strong quadratic tendencies, with the magnitude of the curvature increasing with increasing $\varrho$. The linear term complicates behavior, causing $\varrho$ to increase for small shifts of the curve to the right. Evidently for some $\varrho$ dependent offset, the best fit will coincidentally give the true value of $\varrho$.

\section{Wigner distribution as a two-parameter fit}

In fitting experimental TWDs, it becomes apparent that in many cases - particularly when the data are relatively poor - the CGWDs giving the best fits have first moments different from the first moments of the data. GE noted that the peak of TWDs can be well fitted by treating a Gaussian as a three-parameter fitting function, with the peak position and the prefactor allowed to vary along with the standard deviation. [Presumably the prefactor differs from its expected value, set by normalizing the Gaussian, because of the existence of a small 'hump' sometimes observed at large values of $s$ (see below).] In contrast, it is not clear how such arbitrary modifications could be made to the CGWD, nor is it clear what physical information could be extracted from a CGWD with arbitrary modifications.

From a basic perspective, though, it might be desirable to determine the scaling length (the 'effective mean', which equals the first moment for ideal CGWDs) and the variance in a single fitting procedure rather than to find this length first from the first moment or otherwise. For the following discussion, we denote by $\bar{l}$ the effective mean determined as one parameter of a two-parameter least-squares fit of the data to a CGWD, the other parameter being the exponent $\varrho$. This refined scaling implies that the argument of $P_{\varrho}$ should be $l / \bar{l}$. It is convenient to introduce a new adjustable parameter $\alpha$ which gives the ratio of $\bar{l}$ to the actual 
mean step separation $\langle l\rangle$. Since $s-$ still defined as $l /\langle l\rangle$ - is the natural variable to use in describing data, our refined scaling translates into replacing $s$ by $s / \alpha$ in the argument of the distribution. If the integration variable $s$ were also replaced by $s / \alpha$, then the refined scaling would amount to a redefinition of a dummy variable, and normalization would still be realized. Since the independent variable is kept as $s$, the extra factor is associated with $P(s)$ instead:

$\breve{P}_{Q, \alpha}(s) \equiv \frac{P_{\varrho}(s / \alpha)}{\alpha}$

In other words, the first moment of the distribution, $\mu_{1} \equiv\langle l\rangle$ occurs at $1 / \alpha$ times the optimal characteristic terrace width $\bar{l}$.

We used Mathematica ${ }^{\circledR}$ regression routines to fit the experimental data by minimizing the value of $\chi^{2}$ as a function of the adjustable parameters. Since the values of $s$ are quantized (cf. Section 6), there was assumed to be no error in these values. For simplicity, all data points were weighted equally.

\subsection{Copper: moderately strong repulsion}

Our findings for vicinal $\mathrm{Cu}$ surfaces are presented in Table 1, which is similar to Table 2 of GE, but contains many cases of 'poor' data omitted in GE. In order to facilitate discussion, TWDs were divided by GE into three groups based on a visual assessment of their quality:

- A 'good' TWD changes height essentially monotonically below the peak and again above it; there are no dips, humps, or double peaks, and there is minimal scatter in the data points. 'Good' data are indicated by a ' + ' in Table 1.

- An 'OK' TWD has more scatter, with small dips and peaks introduced by variations (within the limits of the general margin of error) of single data points. 'OK' data are indicated by a ' 0 ' in Table 1.

- A 'poor' TWD had a double-peak or hump at large $s$; correspondingly, the position of the (main) peak occurs noticeably below $s=1$, even when the peak is fairly narrow and the skewness minimal. The judgment that this data is 'poor' is based both on the intuition of the experimenter and on the following argument: a second peak at large $s$ would be characteristic of the onset of faceting; however, 'poor' data tends to occur at high temperatures, whereas faceting should be more important at low temperatures. 'Poor' data are indicated by a '-' in Table 1.

As expected, the Gaussian distribution yields a reasonable, but not exceptional, fit to the data; it worked especially well on surfaces with low temperatures, so relatively large $\tilde{A}$. As an example of good data - exemplified by the vicinal (1 1113$)$ surface at $300 \mathrm{~K}$, depicted in Fig. 1 - the (threeparameter) Gaussian yields a $\chi^{2}$ value of about 0.0072 . The single-parameter CGWD fit gives a slightly worse fit to the data, having a $\chi^{2}$ of 0.0078 . For the two-parameter Wigner fit [Eq. (13)], the $\chi^{2}$ value improves by better than a factor of two, to 0.0037 , with a value of $\varrho$ increased slightly (from 6.4 and 6.5), leading to a value of $\sigma$ closer to that from the Gaussian fit. In this case, the optimal fit using Eq. (13) is obtained by scaling the terrace widths with a value that is $96.5 \%$ of that given by the first moment of the distribution. In other words, the first moment of the TWD is $3.6 \%$ greater than the value of the mean spacing associated with the best fit of the distribution.

In Fig. 2, we display results for this same vicinal $\mathrm{Cu}$ surface at $378 \mathrm{~K}$ as an example of poor data, with a large shift in the effective mean. In this case, having extra degrees of freedom in the fit makes a sizable difference. For the three-parameter Gaussian fit, the $\chi^{2}$ is $0.035 ; \chi^{2}$ increases to 0.042 for the single-parameter Wigner fit and to about half that value, 0.025 , for the two-parameter fit, all these values being half an order of magnitude larger than in the previous, good case. The value of $\varrho$ increases noticeably - from 2.5 to 3.0 - when the refined scaling is allowed (and rises to 4.3 for the shifted-mean method). The refined scaling factor for terrace widths is 0.867 , meaning that the explicit average $\langle l\rangle$ of the TWD is $15.3 \%$ greater than the value of the mean spacing associated with the best fit of the distribution. Characteristic of this sort of data is the hump on the high-s side of the peak, which distorts the single-parameter CGWD fit so that it poorly reproduces the peak region. 


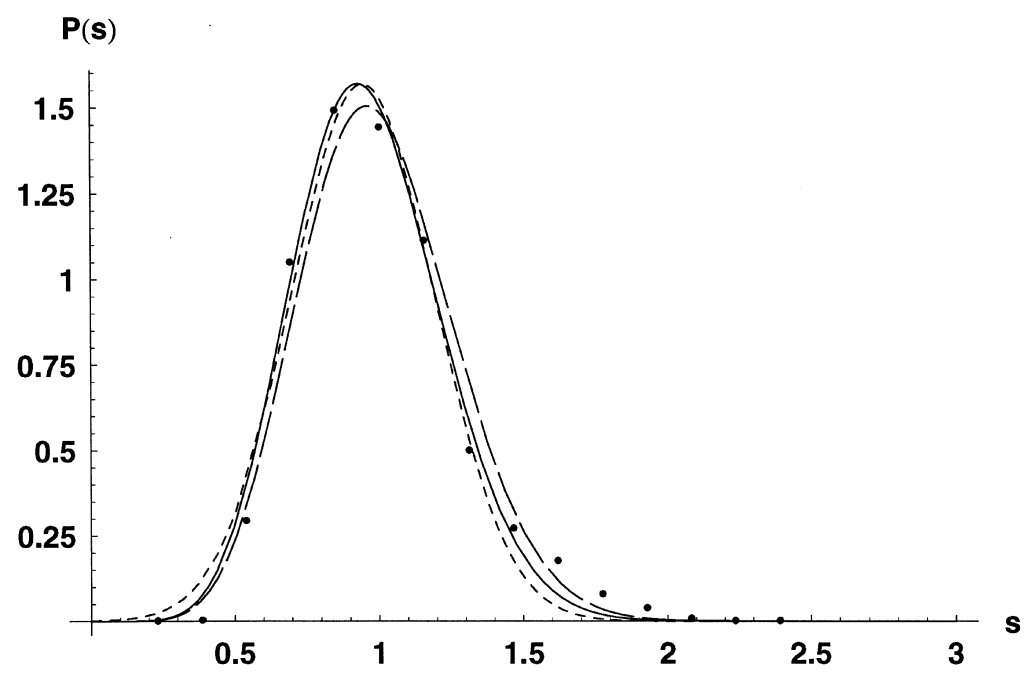

Fig. 1. The vicinal surface $\mathrm{Cu}\left(\begin{array}{lll}1 & 1 & 13\end{array}\right)$ at $300 \mathrm{~K}$ is an example of good data. The points show the normalized data from the STM image. The conventional (three-parameter) Gaussian fit to the data is - - - the fitted standard deviation is $\sigma_{\mathrm{G}}=0.25 \pm 0.01$. The fit to a generalized Wigner distribution with the exponent $\varrho$ as the single adjustable parameter is ——. The best-fit result is $\varrho=6.4 \pm 0.5$, leading via Eq. (4a) to the estimate $\sigma_{\mathrm{W}}=0.26 \pm 0.01$. The terrace widths are scaled by the mean spacing determined from the average of the data. The Wigner distribution is treated as a two-parameter function is - We now find $\varrho=6.4 \pm 0.3$, leading again to $\sigma_{\mathrm{W}}=0.26 \pm 0.01$.

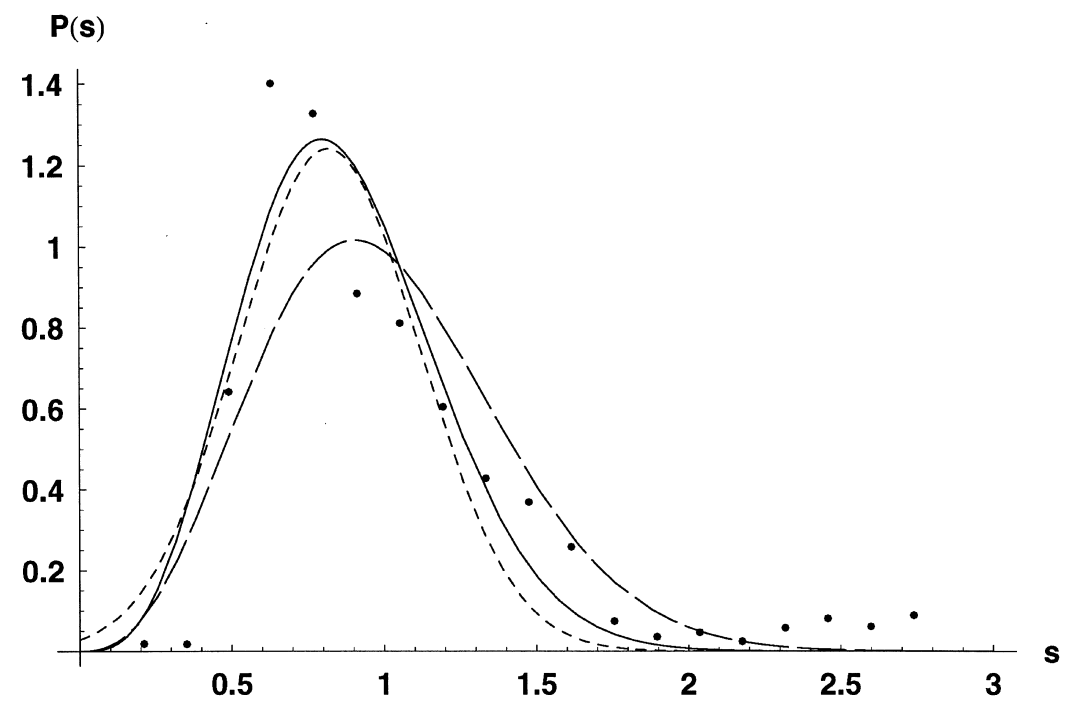

Fig. 2. The vicinal surface $\mathrm{Cu}\left(\begin{array}{lll}1 & 1 & 13\end{array}\right)$ at the higher temperature $378 \mathrm{~K}$ is an example of poor data. As in Fig. 1, $\bullet$, the data; - - -, a conventional Gaussian fit; _ _, a single-parameter Wigner fit; __, a two-parameter Wigner fit. For the Gaussian fit, we get $\sigma_{\mathrm{G}}=0.30 \pm 0.04$, a broader distribution than in Fig. 1 , as expected for the higher temperatures. In contrast to Fig. 1, there is a considerable difference between the two Wigner fits, with the two-parameter version providing a much better accounting due to its ability to adjust to accommodate the points near the peak. For the one-parameter fit, we find $\varrho=2.5 \pm 0.7$, leading to $\sigma_{\mathrm{W}}=0.39 \pm 0.03$, while for the two-parameter fit, we get $\varrho=3.0 \pm 0.5$, leading to $\sigma_{\mathrm{W}}=0.36 \pm 0.03$. The small undulations in the data on the high-s side of the peak, in this example near $s=1.5$ and again for larger $s$, are characteristic of poor data. 
We emphasize the following general trends in Table 1: In almost all instances, the value of $\vec{l}$ derived from the two-parameter fit to a CGWD is smaller than $\mu_{1}=\langle l\rangle$ given by the first moment (the average) of the TWD; likewise, the directly measured values of $\sigma$ are almost always larger than the values obtained by any of the three fitted curves (cf. Section 7). The value of $\varrho$ is higher for the scaled fit than for the single-parameter CGWD fit, and the associated value of $\sigma$ typically closer to that deduced from the Gaussian fit. For 'good' data, the change of value of $\mu$ is of the order of a few percent, and the change in $\varrho$ and $\sigma$ is negligible. For 'poor' data, the refined scaling factor is at least twice as large, and the two-parameter-fit curve is narrower than the single-parameter-fit curve. The tails or humps in the experimental TWDs seem to be responsible for the systematic discrepancies in the fits, especially the smaller mean and smaller variance of the fits relative to the direct measurements.

\subsection{Platinum: weak repulsions}

We have also considered recently reported data for vicinal $\mathrm{Pt}(110)$ at room temperature [6]. In this system the terraces are $(1 \times 2)$ reconstructed, and the steps correspond to three-unit segments [as would be found in a $(1 \times 3)$ reconstruction]. The authors in that paper conclude that the interaction between their steps is small, but are unable to proceed to a quantitative assessment using preexisting methods: Gaussian methods are utterly inappropriate for this regime of small interactions.

In Fig. 3, we show single- and two-parameter Wigner fits of the data. For the former, $\varrho=2.06$ $(\tilde{A}=0.0309)$, with a $\chi^{2}$ of 0.008 . With the latter, the optimal $\bar{l}$ for determining $s$ is $91 \%$ of $\langle l\rangle$ predicted by the average of the data (viz. $\alpha=0.91$ ); $\varrho$ rises to $2.24(\tilde{A}=0.134)$ and the quality of the fit improves to $\chi^{2}=0.003$.

Thus, the high-s bulge does not seem to be peculiar to the vicinal $\mathrm{Cu}$ systems of GE. We do not understand the physical origin of the systematic need for refined scaling of experimental data. We see no comparable effect in our companion Monte Carlo simulations, reported elsewhere [9].

\section{Effects of discreteness on continuum models of a TWD}

Due to the crystalline nature of the surface, the TWD is a discrete rather than a continuous function: the TWD should have a sizable number of counts only at values of that are $a_{\perp}$ times the sum of an integer and a constant fractional offset determined by the terrace and the orientation of the steps (e.g., this offset is $1 / 2$ for close-packed steps on $\left\{\begin{array}{llll}1 & 0 & 0\end{array}\right\}$ surfaces of fcc crystals). For simplicity we neglect this offset in this paper, setting it to zero (as on $\left\{\begin{array}{lll}1 & 0 & 0\end{array}\right\}$ surfaces of sc crystals). Thus, $s$ can only take on the values $s_{L} \equiv L a_{\perp} /\langle l\rangle \equiv L /\langle L\rangle, L$ being a positive integer. It is very tempting simply to apply formulae derived for the continuous TWD given by Eq. (1). In this section we discuss the potential difficulties posed by the discrete nature of the TWD. Inspired by the sealing of discrete TWDs [10], we construct a discrete generalized Wigner distribution (DGWD) TWD given by

$\breve{P}_{\varrho}(s)=\breve{a}_{\varrho} s^{\varrho} \exp \left(-b_{\varrho} s^{2}\right) \sum_{L} \delta\left(s-s_{L}\right)$,

where $\breve{a}_{\varrho}=a_{\varrho} /\langle L\rangle$ is determined by the requirement of normalization.

Although $b_{Q}$ was defined so as to make the mean of the CGWD unity, there is no guarantee that the same parameter will make the mean of the DGWD unity; likewise, the two functions may have different variances. We chose values of $\langle L\rangle$ and $\varrho$ to specify a DGWD and then numerically performed two-parameter fits using CGWD formulae [Eq. (1)-(3), (13)] to produce estimates of $\varrho_{\text {c }}$. Anticipating greater interest in behavior as a function of $\tilde{A}$ than of $\varrho$, we converted our results for $\varrho_{\mathrm{c}}$ to $\tilde{A}$ using Eq. (3).

Fig. 4 shows the difference of the fitted value $\tilde{A}_{\mathrm{c}}$ and the 'parent' value $\tilde{A}$ as a function of this $\tilde{A}$ for several mean widths $\langle L\rangle$. As may be expected, as the TWD becomes narrower (i.e. for sufficiently large $\tilde{A}$ or $\varrho$ ), $\tilde{A}_{\mathrm{c}}$ becomes a decidedly unreliable estimate for $\tilde{A}$; based on examination of the cases $\langle l\rangle / a_{\perp}=2-6$, this breakdown appears to occur for $\varrho$ near $\langle L\rangle^{2}$. This threshold corresponds to $s_{L+1}-s_{L} \equiv a_{\perp} /\langle l\rangle \equiv\langle L\rangle^{-1}=\sigma$. Thus, for $\langle L\rangle<4$, this breakdown occurs to the region of physical interest (cf. dashed curves in Fig. 4). On 


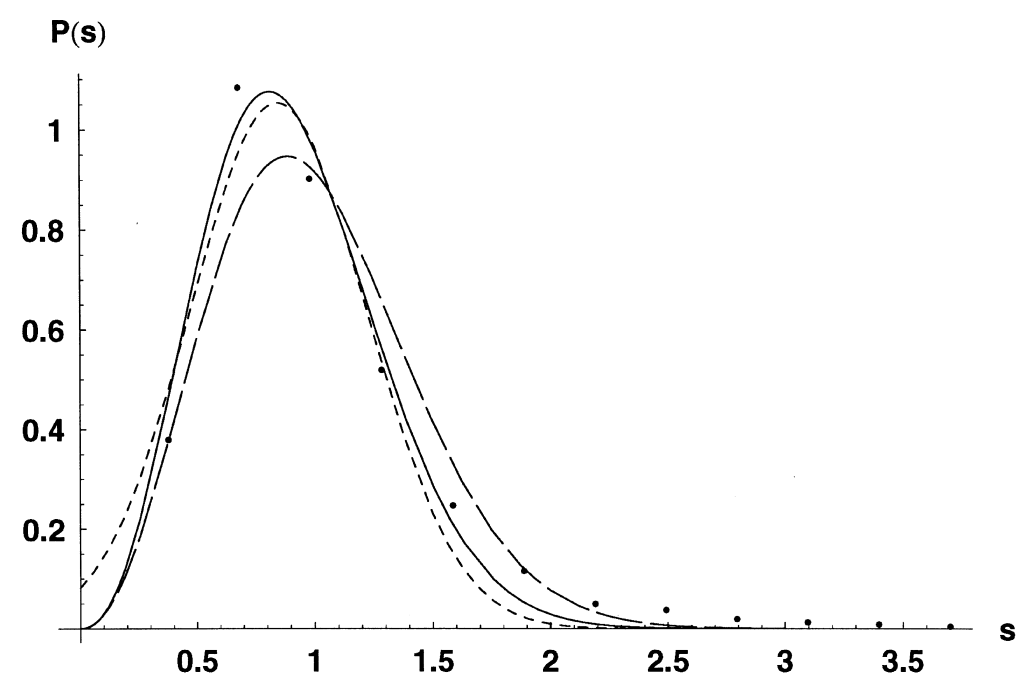

Fig. 3. Analysis of TWDs of Pt(110) using Wigner distributions. -, Experimental points of Ref. [6]. As in Figs. 1 and 2, ---, conventional Gaussian fit; _ _ , single-parameter Wigner fit; __ , a two-parameter Wigner fit.

the other hand, for $\langle L\rangle \geq 4, \tilde{A}_{\mathrm{c}}$ provides a reasonable estimate of $\tilde{A}$ over the range of physically reasonable dimensionless repulsions, where the effects of discreteness are most pronounced for

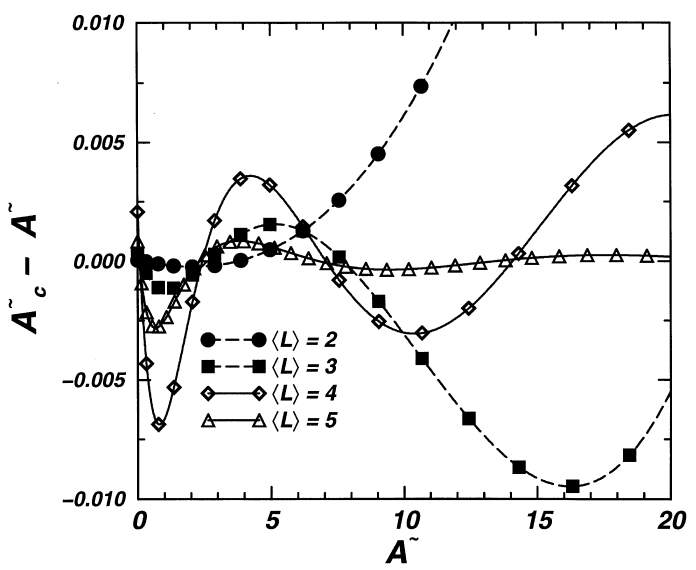

Fig. 4. The error in estimates $\tilde{A}_{c}$ of $\tilde{A}$ derived by using formulae for the mean and variance of the continuous generalized Wigner surmise TWD on discrete TWDs, for the physical range of $\tilde{A}$. $\langle L\rangle$ indicates the mean terrace width in units of $a_{\perp}$. For $\langle L\rangle=$ 2 and 3 , the ordinate values have been divided by 1000 and by 50 , respectively, to appear on the same vertical scale; evidently discreteness for these narrow terraces introduces unacceptably large errors, particularly as $\tilde{A}$ increases. The smooth curves through these points, to guide the eye, are dashed to distinguish them from the cases with broader terraces. small values of $\langle L\rangle$ of $\tilde{A}$. Note also that in each case there is a substantial peak in $\left|\tilde{A}_{\mathrm{c}}-\tilde{A}\right|$ for small $\tilde{A}$. Fig. 5 shows the reduction in the error in $\tilde{A}_{\mathrm{c}}$ as $\langle L\rangle$ increases, at fixed values of $\tilde{A}$.

In summary, we have raised a flag of caution when analyzing the fluctuations of highly misoriented vicinal surfaces in a conventional framework. The case of $\langle L\rangle=3$ corresponds to $(1,1,7)$ for

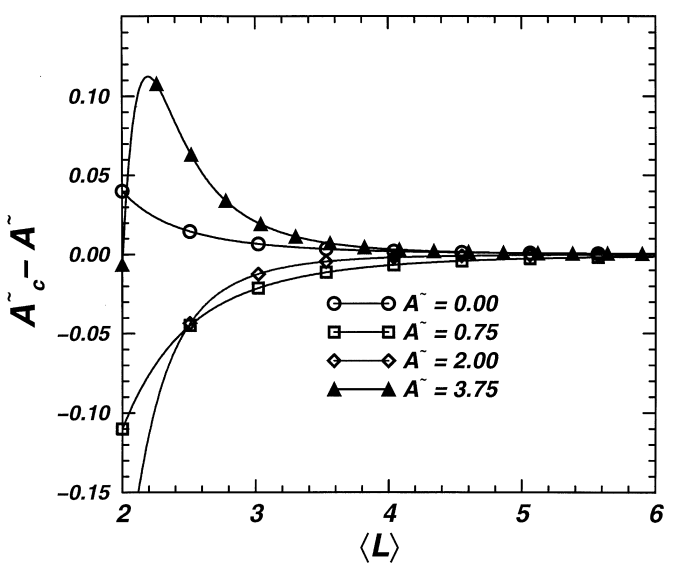

Fig. 5. The error in estimates $\tilde{A}_{c}$ of $\tilde{A}$ derived by using formulae for the mean and variance of the continuous generalized Wigner surmise TWD on discrete TWD. The estimates evidently improve considerably with increasing $\langle L\rangle$ (broader terraces, with higher Miller indices). 
close-packed steps on surfaces vicinal to $\left\{\begin{array}{lll}1 & 0 & 0\end{array}\right\}$ planes of fcc crystals. Thus, one should view with some suspicion the unusual large values of $\varrho$ and $\tilde{A}$ reported for the single temperature at which this vicinal $\mathrm{Cu}$ surface was measured. For $\left\{\begin{array}{lll}1 & 1 & 1\end{array}\right\}$ surfaces, the corresponding Miller indices are $\left(\begin{array}{llll}5 & 3 & 3\end{array}\right)$ for A steps ( $\left\{\begin{array}{lll}1 & 0 & 0\end{array}\right\}$ microfacets) and (2 21$)$ for B

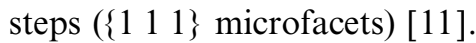

We also emphasize that this behavior is not a vagary of Wigner distributions. Misorientation causes similar problems when the mean and variance of discretized Gaussian TWDs are analyzed as though they were continuous Gaussian functions. For more convenient comparison with the above Wigner distribution, we used Eq. (4b) to relate the variances and values of $\varrho$. We found that estimates of $\varrho$ based on the variance of the discretized Gaussians approached the undiscretized value monotonically, rather than oscillating as in the case of the Wigner distribution, and that the approach to the undiscretized value of $\varrho$ is actually somewhat slower in the Gaussian case than in the Wigner case. The Gaussian case also showed a breakdown at large values of $\varrho$ (small $\sigma^{2}$ ) similar to the Wigner case.

\section{Statistical uncertainties due to finite sampling size}

By truncating Eq. (6) at the second term, we can create an estimator $\hat{\varrho}$ for $\varrho$ :

$\hat{\varrho}=\frac{1}{2}\left({\widehat{\sigma^{2}}}^{-1}-\frac{3}{4}\right.$,

where $\widehat{\sigma^{2}}$ is a random variable that is an estimator of $\sigma^{2}$. For small $\sigma^{2}$, though, the Wigner distribution approaches a more familiar Gaussian distribution, as discussed in Section 4. For a Gaussian distribution, the sampling errors from a sample of size $N_{\text {samp }}$ for $\widehat{\sigma^{2}}$ are given by [12]:

$\operatorname{var}\left(\widehat{\left.\sigma^{2}\right)}=\frac{2 \sigma^{4}}{\left(N_{\text {samp }}-1\right)}\right.$.

Accordingly, the standard deviation of the estimated values of $\varrho$ can be seen to increase with increasing values of $\varrho$ :

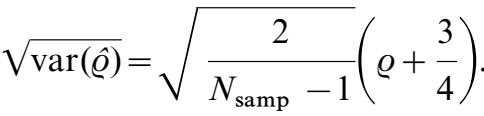

In this section we explore the effects of statistical fluctuations on the estimated value of $\varrho$ by performing some well-defined numerical experiments. The results are thus applicable to 'ideal' data. In fact there apparently are systematic effects, noted earlier, in real data that limit the applicability of some deductions.

Specifically, we begin with the following simple procedure: First, we independently choose $N_{\text {samp }}$ values of $s$ using the same known DGWD as the probability density function for each selection. Second, we fit this artificial TWD using the twoparameter Wigner distribution $\breve{P}_{\mathrm{e}, \alpha}(s)$ to determine $\varrho$, taking each point to be weighted equally in accordance with standard practice [2]. Third, we repeat this process a large number of times and measure the standard deviation of the fitted values of $\varrho$ as well as any systematic bias in the fitted estimates.

Fig. 6 shows the result of this procedure, with one million independent TWDs produced for each value of $\varrho$, and each TWD consisting of 801 independent values of $s$ drawn according to a DGWD. Clearly the linear relationship between the $\sqrt{\operatorname{var}(\hat{\varrho})}$ is maintained, but the slope is somewhat larger than predicted by Eq. (17).

Another way of estimating $\varrho$ is to measure directly the mean and variance of the TWD and to insert them into Eq. (6). Repeating our procedure of creating artificial TWDs, we accordingly estimate $\varrho$ using Eq. (6), again analyzing the variance of the estimates as above. As seen in Fig. 6, the resulting estimates of $\varrho$ have variances given almost exactly by Eq. (17) and noticeably smaller (though not by a large factor) than the variances given by the traditional, uniformlyweighted nonlinear least-squares fits. This finding means that not only is it possible to use simple analytic functions to find $\varrho$ and $\tilde{A}$ instead of using two-parameter nonlinear least-squares fits, but also that doing so is statistically better!

This result appears to be contrary to the belief that performing a least-squares fit to an appro- 


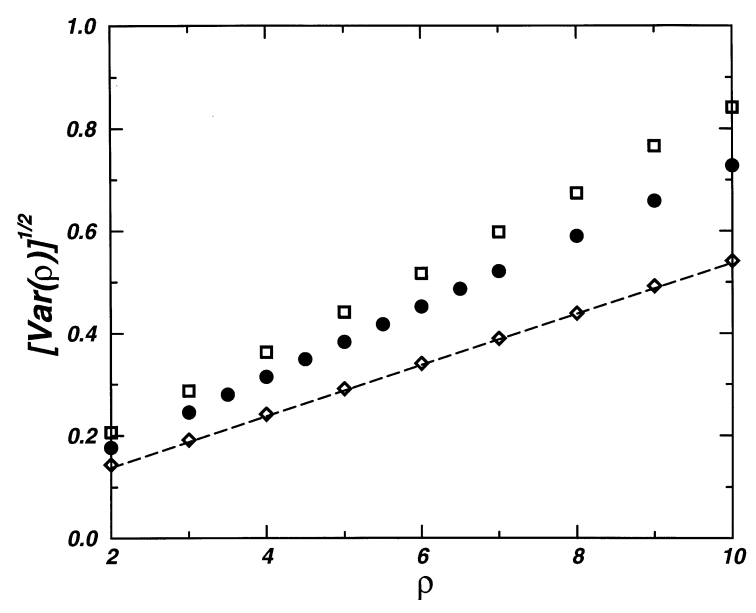

Fig. 6. Standard deviations in fitted values of $\varrho$ due to statistical fluctuations. In each case, fits were made to TWDs consisting of $N_{\text {samp }}=801$ values of $s$ independently distributed according to a DGWD function. Each circle represent a sampling over one million uniformly-weighted two-parameter fits. Each square represents a sampling over ten thousand two-parameter fits, in which the weight for each point in the TWD was weighted proportionally to $P(s)$. Each diamond represents a sampling over ten thousand applications of Eq. (6). The line is the prediction of Eq. (17). Both in terms of computational difficulty and statistical quality, Eq. (6) is clearly superior to nonlinear leastsquares fits.

priate smooth function is desirable to minimize the effects of statistical fluctuations. It seems likely, though, that the real problem lies in the weighting of the data in the fit. It has been suggested that greater weight should be given to the points near the peak of the TWD, so we once again repeat our procedure, this time making a least-squares fit in which each point is weighted proportionally to the measured value of $P$. As Fig. 6 shows, the standard deviation of $\varrho$ again varies linearly with $\varrho$, but with a slope that is slightly higher than that of the uniformly-weighted case. In retrospect, this result should not be surprising, since each point on the TWD represents the result of $N_{\text {samp }}$ binomial experiments (i.e. Bernoulli trials: either the measurement of step separation gives this distance $s_{L}$ or some other distance). Elementary statistics [13] shows that the statistical error of binomial experiments is smallest when the probability of success is nearly zero or nearly one - in our case, for points on the TWD with $P(s) \approx 0$. However, devising a naïve weighting by the reciprocal of the variance of each point on the TWD is problematic when points for which the measured value of $P(x)$ is equal to zero; these points would receive infinite weight, yielding nonsensical results. Even if one circumvents this problem, there is still the problem that the points are not uncorrelated, which is a requirement of the least-squares procedure [14]; the normalization condition imposes a (weak) correlation between points. We can avoid these problems by simply using the mean and variance of the TWD in Eq. (6) to find $\varrho$ or Eq. (7) to find $\tilde{A}$.

Motivated by these numerical experiments, we computed directly (from the histogram data, after normalization and adjustment to unit mean) the standard deviation $\sigma_{\text {dir }}$ (cf. Table 1). This result is systematically higher than the $\sigma$ 's obtained from the various fitting techniques. The difference is modest for good data but pronounced for poor data. Thus, as mentioned in Section 5, it appears to come from the curious high-s undulations that plague poor data. The fitting techniques, being less sensitive to these points, give values of $\sigma$ that are less distorted by them.

Finally, we note that single STM images do not allow for a large number of independent values of $s$. The number of independent measurements is generally much smaller than the total number of measurements, due to correlations between the measurements. Although a precise determination of the effects of correlations on fitted parameters would be rather involved, a working estimate of the number of 'independent' measurements - from which uncertainties can be estimated - can be made in the following way. First, one obtains the terrace width $l_{n}(y)$ between steps $n$ and $n+1$ for each position $y$ along the steps. Then the correlation function [14]

$$
\begin{aligned}
& C_{n}(y) \equiv \frac{\left\langle l_{0}(0) l_{n}(y)\right\rangle-\langle l\rangle^{2}}{\left\langle l^{2}\right\rangle-\langle l\rangle^{2}} \\
& =\frac{1}{\left\langle l^{2}\right\rangle-\langle l\rangle^{2}}\left[\frac{\sum_{n^{\prime}}^{N-n} \sum_{y^{\prime}=1}^{L_{y}-y} l_{n^{\prime}}\left(y^{\prime}\right) l_{n^{\prime}+n}\left(y^{\prime}+y\right)}{(N-n)\left(L_{y}-y\right)}-\langle l\rangle^{2}\right]
\end{aligned}
$$


is calculated, where $N$ is the number of terraces in the image (i.e. $N+1$ is the number of steps). The correlation function along the steps decays exponentially as $C_{0}(y) \sim \exp \left(-y / \xi_{y}\right)$, where $\xi_{y}$ is the intrastep correlation length. ${ }^{2} \xi_{y}$ is given by Eq. (18) of Ref. [15], but the safest procedure is simply to measure it. The correlation function between steps, on the other hand, is more complicated; $C_{1}(0)$ is negative [3], but the trend is for the absolute value of $C_{n}(0)$ to decrease rapidly with increasing $n$. We define $y_{c}$ to be the smallest value of $y$ for which

$\left|C_{0}(y)\right| \leqslant c \quad \forall y \geqslant y_{c}$,

and likewise $n_{c}$ to be the smallest value of $n$ for which

$\left|C_{n}(y)\right| \leqslant c \quad \forall n \geqslant n_{c}$,

where $c$ is a small cutoff (we recommend $c=0.1$ ). The number of 'independent' terrace widths will be approximately $\left(L_{y} / y_{c}\right)\left(N / n_{c}\right)$.

As an example, we performed a Monte Carlo simulation of a system with $A=0$. For simplicity, we chose $k_{\mathrm{B}} T$ to be equal to the energy for producing a kink, and we chose the mean distance between steps to be ten lattice units. We measured $\xi_{y}=15$ (consistent with theoretical predictions, see preceding footnote) and $y_{c}=40$, and we observed that $\left|C_{n}(0)\right| \leq 0.1$ for all $n \geq 3$. Suppose this had been an STM image representing a square region of the crystal 200 lattice units on a side; then there would be approximately 20 terraces in the image, and $(200 / 40)(20 / 2)=50$ independent widths much smaller than the 4000 independent measurements that one might naively suspect. As a result, we see that the uncertainty in statistically derived quantities such as the measured value of $A$ are an order of magnitude larger than the naïve estimate. Lowering the temperature relative to the kink creation energy would have the effect of further reducing the number of independent measurements

\footnotetext{
${ }^{2}$ As discussed in Ref. [15], from the Gruber-Mullins [16] perspective $\xi_{y}=8\langle l\rangle^{2} \tilde{\beta} / 3 \pi^{2} k_{\mathrm{B}} T$ for $A=0$ and $\xi_{y}=2\langle l\rangle \tilde{\beta} /$ $k_{\mathrm{B}} T$ for $A \gg 0$.
}

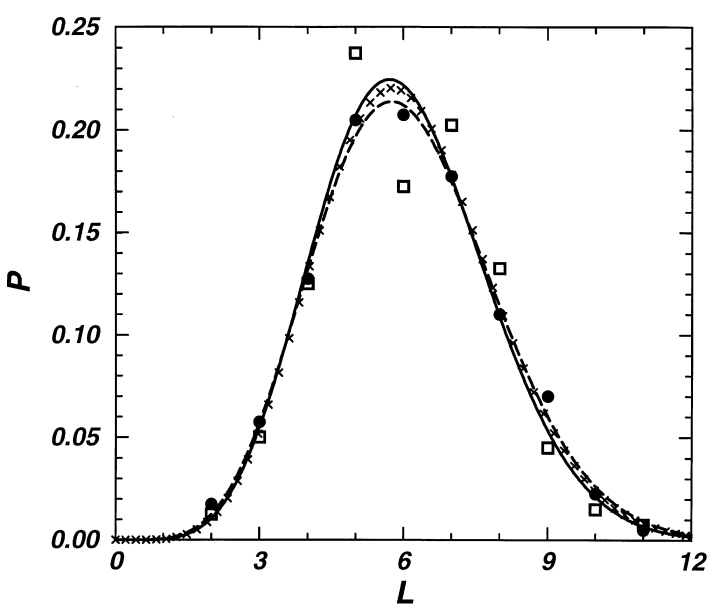

Fig. 7. Twenty TWDs were simulated by drawing $N_{\text {samp }}=400$ values of $s$ according to a DGWD with $\varrho=5$ and $\langle L\rangle=6$, indicated by the solid curve. Each TWD was then fitted to a singleparameter CGWD, as in Eqs. (1)-(3). Shown are the TWDs with the smallest $(\bullet)$ and largest $(\square)$ values of $\chi^{2}$. The fits to these are shown as the black dashed curve and the $\times \mathrm{s}$, respectively.

and thus increasing the uncertainty in the measured value of $A$.

With such small samples, the measured TWD can differ distinctly from the DGWD due to statistical fluctuations alone. In order to demonstrate this idea, we produce 20 TWDs, each consisting of 400 independent values of $s=s_{\mathrm{L}}$ sampled from a DGWD with $\varrho=5$ and $\langle L\rangle=6$, and fitted each TWD with a DGWD. (In this model, $s_{L}=L /\langle L\rangle$; there is no offset between successive terraces.) Fig. 7 shows the TWDs with the lowest and the highest values of $\chi^{2}$. Curiously, in this particular case the TWD with the largest value of $\chi^{2}$ happens to produce better estimates of both $\varrho$ and $\langle L\rangle$ than does the TWD with the smallest value of $\chi^{2}$. In no case, however, do we see the shoulders or second peaks in the TWD at large values of $s$ occur systematically in the 'poor' data of Fig. 2 here or Fig. 5b in Ref. [2]. Since the 'poor' data were based on several dozen independent STM measurements, they should be statistically comparable to the data of Fig. 7, but the systematic deviation indicates that the 'poor' data cannot be entirely understood within the framework of a generalized Wigner distribution. 


\section{Conclusions}

In this paper we have performed several numerical experiments and analyses to understand better the TWDs derived from physical data from vicinal surfaces. We have quantitatively studied how the Wigner distribution approaches a Gaussian for large dimensionless interactions, and shown that for most systems of physical interest the standard deviation of the terrace width can be estimated from either distribution with little difference.

The mean step width can be estimated in a variety of ways for both Wigner and Gaussian distributions; two reasonable but inequivalent choices are directly averaging the step width and fitting the TWD to the desired distribution using a nonlinear least-squares routine. In Section 4 we discuss the effects of using an estimated mean that differs from the least-squares estimate. On the other hand, the adjustment of the normalization of the curve to obtain a 'better' fit is unjustified; even if the visual agreement appears to improve, no results from theory - such as interaction strengths - can be meaningfully extracted from TWDs with the wrong normalization.

We have proposed a two-parameter extension of the generalized Wigner surmise, which really is just a consistent fitting of both $\varrho$ and the mean terrace width within a single two-parameter leastsquares fit. This added flexibility allows one to deal more fruitfully with poorer-than-desirable experimental data, while not changing good data (or the data emerging from various numerical simulations). Thus, this fitting function can be applied generally. On the other hand, for 'good' data, we have shown that a simple series expansion based on the directly measured mean and standard deviation of the terrace widths has better statistical properties. The least-squares fit is more robust, but this is a property that is useful only when the Wigner distribution does not capture all the important physics, such as the role of defects or more complicated step-step interactions; in such cases, the estimated values of the step-step interaction from the Wigner - or the Gaussian - distribution must be treated with great caution.

Finally, we emphasize the importance of using many STM measurements to ensure good statistics and the desirability of calculating the correlations between terrace widths within individual STM images. As we saw in Section 7, the typical STM image suitable for measuring terrace widths will contain no more than about 50 independent terrace width measurements, almost two orders of magnitude less than the 4000 total terrace width measurements.

\section{Acknowledgements}

Work at Maryland was supported by NSFMRSEC Grant No. DMR-9632521 and benefited from interactions with E.D. Williams. SDC, in particular, participated in an NSF-MRSEC-sponsored REU program. The authors are grateful to K. Swamy and E. Bertel for sending their data for vicinal $\mathrm{Pt}(110)$. TLE also acknowledges the support of a Humboldt U.S. Senior Scientist Award and the hospitality of the IGV at FZ-Jülich.

\section{Appendix A: Derivation of expansions}

In this appendix we derive a useful series expansion Eqs. (2) and (8) for the coefficient $b_{\varrho}$ in the quadratic exponential of the generalized Wigner distribution. For convenience, we define the variable

$r \equiv \frac{\varrho+1}{2}$

Then we use Stirling's asymptotic series [17], $\Gamma(r)=\pi^{1 / 2} \exp (-r)^{r-1 / 2} F(r)$ :

$$
\begin{aligned}
b_{\varrho} & \equiv\left[\frac{\Gamma(r+1 / 2)}{\Gamma(r)}\right]^{2} \\
& =\left[\frac{e^{-(r+1 / 2)}(r+1 / 2)^{r} F(r+1 / 2)}{e^{-r}(r)^{r-1 / 2} F(r)}\right]^{2},
\end{aligned}
$$

where

$$
\begin{aligned}
F(r)= & +\frac{1}{12 r}+\frac{1}{288 r^{2}}-\frac{139}{51840 r^{3}} \\
& -\frac{571}{2488320 r^{4}}+\mathcal{O}\left(r^{-5}\right) .
\end{aligned}
$$


We concentrate initially on the first part of the fraction:

$\left[\frac{e^{-(r+1 / 2)}(r+1 / 2)^{r}}{e^{-r}(r)^{r-1 / 2}}\right]^{2}=e^{-1} r\left(1+\frac{1}{2 r}\right)^{2 r}$.

But

$$
\begin{aligned}
(1+ & \left.\frac{1}{2 r}\right)^{2 r}=\exp \left\{\ln \left[\left(1+\frac{1}{2 r}\right)^{2 r}\right]\right\} \\
= & \exp \left\{\left[1-\frac{1}{2}(2 r)^{-1}+\frac{1}{3}(2 r)^{-2}-\frac{1}{4}(2 r)^{-3}\right.\right. \\
& \left.\left.+\frac{1}{5}(2 r)^{-4}+\mathcal{O}\left[(2 r)^{-5}\right]\right]\right\} \\
= & \mathrm{e}^{+1}\left[1-\frac{1}{2}(2 r)^{-1}+\frac{11}{24}(2 r)^{-2}-\frac{7}{16}(2 r)^{-3}\right. \\
& \left.+\frac{2477}{5760}(2 r)^{-4}+\mathcal{O}\left[(2 r)^{-5}\right]\right] .
\end{aligned}
$$

It is also straightforward to show that

$$
\begin{aligned}
{\left[\frac{F(r+1 / 2)}{F(r)}\right]^{2}=} & 1-\frac{1}{3}(2 r)^{-2}+\frac{1}{3}(2 r)^{-3} \\
& -\frac{13}{90}(2 r)^{-4}+\mathcal{O}\left[(2 r)^{-5}\right] .
\end{aligned}
$$

By combining all of these we find

$$
\begin{aligned}
b_{\varrho}= & r\left(1-\frac{1}{4}+\frac{1}{32} r^{-1}+\frac{1}{128} r^{-2}\right. \\
& \left.-\frac{13}{6144} r^{-3}+\mathcal{O}\left[r^{-4}\right]\right) .
\end{aligned}
$$

From this formula for $b_{\varrho}$, we can use Eq. (4a) to write $\sigma^{2}$ as a power series in $\varrho^{-1}$ :

$$
\begin{aligned}
\sigma^{2}= & \frac{1}{2}(\varrho+1)^{-1}+\frac{1}{8}(\varrho+1)^{-2}-\frac{1}{16}(\varrho+1)^{-3} \\
& -\frac{17}{384}(\varrho+1)^{-4}+\mathcal{O}\left[(\varrho+1)^{-5}\right] \\
= & \frac{1}{2} \varrho^{-1}-\frac{3}{8} \varrho^{-2}+\frac{3}{16} \varrho^{-3}+\frac{7}{384} \varrho^{-4}+\mathcal{O}\left[\varrho^{-5}\right] .
\end{aligned}
$$

Using reversion of series, we then find

$$
\varrho^{-1}=2 \sigma^{2}+3\left(\sigma^{2}\right)^{2}+6\left(\sigma^{2}\right)^{3}+\frac{32}{3}\left(\sigma^{2}\right)^{4}+\mathcal{O}\left[\left(\sigma^{2}\right)^{5}\right],
$$

from which we get

$$
\begin{aligned}
\varrho= & \frac{1}{2}\left(\sigma^{2}\right)^{-1}\left\{1+\frac{3}{2}\left(\sigma^{2}\right)+3\left(\sigma^{2}\right)^{2}\right. \\
& \left.+\frac{16}{3}\left(\sigma^{2}\right)^{3}+\mathcal{O}\left[\left(\sigma^{2}\right)^{4}\right]\right\}^{-1} \\
= & \frac{1}{2}\left(\sigma^{2}\right)^{-1}\left\{1-\frac{3}{2}\left(\sigma^{2}\right)-\frac{3}{4}\left(\sigma^{2}\right)^{2}\right. \\
& \left.+\frac{7}{24}\left(\sigma^{2}\right)^{3}+\mathcal{O}\left[\left(\sigma^{2}\right)^{4}\right]\right\} .
\end{aligned}
$$

Finally, with Eq. (5) we can use these results to find the dimensionless interaction constant $\tilde{A}_{\mathrm{W}}$ in terms of $\sigma^{2}$ :

$$
\begin{aligned}
\tilde{A}_{\mathrm{W}}= & \frac{1}{16}\left(\sigma^{2}\right)^{-2}-\frac{7}{16}\left(\sigma^{2}\right)^{-1}+\frac{27}{64} \\
& +\frac{35}{96}\left(\sigma^{2}\right)+\mathcal{O}\left[\left(\sigma^{2}\right)^{2}\right] .
\end{aligned}
$$

For $\tilde{A} \geq 0.0525$, the relative error in Eq. (A11) is $<1 \%(<0.1 \%$ for $\tilde{A} \geq 0.15)$. The absolute error is $<1 \%$ for $\tilde{A} \geq-1 / 4$.

\section{Appendix B: Effect of displacement of a Gaussian fitting function in fits of a Gaussian}

Suppose we have a Gaussian distribution with mean $\mu$ and variance $\sigma^{2}$; we attempt to fit this Gaussian with a second Gaussian with a mean $\mu+\Delta \mu$ and a variance $(1-\zeta)^{2} \sigma^{2}$, where $\Delta \mu$ is fixed and $\zeta$ is unknown. Explicitly, we write $\chi^{2}$ as a function of $\zeta$ and $\Delta \mu$ :

$$
\begin{aligned}
\chi^{2}= & \int_{-\infty}^{\infty} \mathrm{d} x\left\{\left[\frac{1}{\sigma(1-\zeta) \sqrt{2 \pi}} \exp \left(-\frac{(x-\mu)^{2}}{2 \sigma^{2}(1-\zeta)^{2}}\right)\right]\right. \\
& \left.-\left[\frac{1}{\sigma \sqrt{2 \pi}} \exp \left(-\frac{[x-(\mu+\Delta \mu)]^{2}}{2 \sigma^{2}}\right)\right]\right\}^{2} \\
= & \frac{1}{2 \sigma(1-\zeta) \sqrt{\pi}}+\frac{1}{2 \sigma \sqrt{\pi}}+\frac{1}{\sigma \sqrt{2 \pi\left[1+(1-\zeta)^{2}\right]}}
\end{aligned}
$$




$$
\begin{aligned}
& \times \exp \left[-\frac{(\Delta \mu)^{2}}{2 \sigma^{2}\left\{1+(1-\zeta)^{2}\right\}}\right] \\
= & \frac{1}{\sigma \sqrt{\pi}}\left(\left[\left(\frac{\Delta \mu}{2 \sigma}\right)^{2}+\mathcal{O}\left\{\left(\frac{\Delta \mu}{2 \sigma}\right)^{4}\right\}\right]\right. \\
+ & {\left[\frac{3}{2}\left(\frac{\Delta \mu}{2 \sigma}\right)^{2}+\mathcal{O}\left\{\left(\frac{\Delta \mu}{2 \sigma}\right)^{4}\right\}\right] \zeta } \\
& \left.+\frac{3}{8}\left[1+3\left(\frac{\Delta \mu}{2 \sigma}\right)^{2}+\mathcal{O}\left\{\left(\frac{\Delta \mu}{2 \sigma}\right)^{4}\right\}\right] \zeta^{2}+\mathcal{O}\left(\zeta^{3}\right)\right) .
\end{aligned}
$$

Since the optimum fit is found by minimizing $\chi^{2}$, we set $\mathrm{d} \chi^{2} / \mathrm{d} \zeta=0$ in Eq. (B1) and remove the overall prefactor $1 /(\sigma \sqrt{\pi})$ to get:

$$
\begin{aligned}
0 & =\left[\frac{3}{2}\left(\frac{\Delta \mu}{2 \sigma}\right)^{2}+\mathcal{O}\left\{\left(\frac{\Delta \mu}{2 \sigma}\right)^{4}\right\}\right] \\
& +\frac{3}{4}\left[1+3\left(\frac{\Delta \mu}{2 \sigma}\right)^{2}+\mathcal{O}\left\{\left(\frac{\Delta \mu}{2 \sigma}\right)^{4}\right\}\right] \zeta+\mathcal{O}\left(\zeta^{2}\right) .
\end{aligned}
$$

Solving for $\zeta$, we find

$$
\zeta=2\left(\frac{\Delta \mu}{2 \sigma}\right)^{2}+\mathcal{O}\left\{\left(\frac{\Delta \mu}{2 \sigma}\right)^{4}\right\} .
$$

Since $\Delta \sigma^{2} / \sigma^{2}=2 \Delta \sigma / \sigma$, Eq. (B2) leads to Eq. (11).

\section{References}

[1] T.L. Einstein, O. Pierre-Louis, Surf. Sci. 424 (1999) L299.

[2] M. Giesen, T.L. Einstein, Surf. Sci 449 (2000) 191.

[3] M.L. Mehta, Random Matrices, second edn, Academic, New York, 1991.

[4] T. Guhr, A. Müller-Groeling, H.A. Weidenmüller, Phys. Rep. 299 (1998) 189.

[5] H.L. Richards, T.L. Einstein, Bull. Am. Phys. Soc. 45 (2000) M31.06, and in press.

[6] K. Swamy, E. Bertel, I. Vilfan, Surf. Sci. 425 (1999) L369.

[7] Y.C. Huang, J. Flidr, T.A. Newton, M.A. Hines, J. Chem. Phys. 109 (1998) 5025.

[8] B. Sutherland, J. Math. Phys. 12 (1971) 246.

[9] S.D. Cohen, H.L. Richards, T.L. Einstein, Bull. Am. Phys. Soc. 45 (2000) M31.05, and in press.

[10] B. Joós, T.L. Einstein, N.C. Bartelt, Phys. Rev. B 43 (1991) 8143.

[11] D.R. Eisner, T.L. Einstein, Surf. Sci. 286 (1993) L559.

[12] P. Armitage, G. Berry, Statistical Methods in Medical Research, second edn, Blackwell Scientific, Boston, 1987, pp. $85-88$.

[13] D.D. Wackerly, W. Mendenhall, R.L. Schaeffer, Mathematical Statistics with Applications, fifth edn, PWS-Kent, Boston, 1996.

[14] G.E.P. Box, W.G. Hunter, J.S. Hunter, Statistics for Experimenters: An Introduction to Design, Data Analysis, and Model Building, Wiley, New York, 1978.

[15] N.C. Bartelt, T.L. Einstein, E.D. Williams, Surf. Sci. 240 (1990) L591.

[16] E.E. Gruber, W.W. Mullins, J. Phys. Chem. Solids 28 (1967) 875.

[17] M. Abramowitz, I.A. Stegun, Handbook of Mathematical Functions, Dover, New York, 1968. 